\title{
OPEN BOUNDARIES IN SHORT WAVE SIMULATIONS - A NEW APPROACH
}

\author{
JESPER LARSEN and HENRY DANCY* \\ Danish Hydraulic Institute, Agern Allé 3, DK-2970 Horsholm (Denmark)
}

(Received September 10, 1982; revised and accepted April 7, 1983)

\section{ABSTRACT}

Larsen, J. and Dancy, H., 1983. Open boundaries in short wave simulations - a new approach. Coastal Eng., 7: 285-297.

A new way of implementing radiation boundary conditions in finite difference schemes is reported. Instead of prescribing the incident field at the model boundary, waves are generated inside the model boundary. All outgoing waves are absorbed at open boundaries using so-called "sponge" layers.

\section{INTRODUCTION}

In the simulation of short waves for coastal engineering applications, the domain of integration is virtually infinite. However, economical constraints necessitate that the computational domain is limited as far as possible. At the open boundary so introduced, proper boundary conditions must account for the coupling between the wave field on the computational mesh and the field in the infinite domain left out of the computation. The standard way of solution is to reformulate the scattering problem as a radiation problem (Israeli and Orszag, 1981). All waves are then outgoing waves and they must be absorbed on open boundaries.

Perfect absorption can only be obtained in very specialized cases, e.g. one-dimensional, linear wave problems (Verboom et al., 1982). For more general problems, higher-order boundary conditions can be found by the use of an expansion technique and the application of pseudo-differential operators (Engquist and Majda, 1977). Another approach is to introduce artificial damping in the formulation of the problem. Israeli and Orszag (1981) have shown how a combination of damping or "sponge" layers and absorbing boundary conditions very efficiently absorbs outgoing waves. Madsen (1983) describes the absorption properties of porous structures in short-wave simulations.

*Stagiaire from Ecole Nationale des Ponts et Chaussées, 28 rue des Saint Pères, F-75007 Paris, France. 
In this paper we report a new way of reformulating the scattering problem as a radiation problem. The incident wave field is simply added on a line inside the computational domain. We consider the implementation of this technique in a finite-difference formulation. Then a very efficient method of implementing "sponge" layers in difference schemes is described, and a linear analysis of the one-dimensional, modified Preissmann box scheme (Preissmann, 1961; McCowan, 1978) is carried out. Finally, a number of results with a two-dimensional scheme (Abbott, 1979) is shown.

\section{THE RADIATION PROBLEM}

In Fig. 1 we show a definition sketch of the problem. Abbott et al. (1978) have shown the feasibility of using the Boussinesq equations for short-wave simulations in shallow water. The equations read (Peregrine, 1967):

$\frac{\partial h}{\partial t}+\nabla \cdot(h u)=0$

and

$\frac{\partial u}{\partial t}+(u \cdot \nabla) u+g \nabla \zeta=\frac{1}{2} D \frac{\partial}{\partial t} \nabla[\nabla \cdot(D u)]-\frac{1}{6} D^{2} \frac{\partial}{\partial t} \nabla(\nabla \cdot u)$

where $h=D+\zeta$ is the water depth, $D$ is the still-water depth, $\zeta$ is the surface elevation and $u=(u, v)$ is the horizontal velocity vector. At solid boundaries the normal velocity should be zero. At open boundaries the incident field $\left(\zeta^{\mathrm{I}}, u^{\mathrm{I}}\right)$ is specified and the scattered field, $\zeta^{\mathrm{S}}=\zeta-\zeta^{\mathrm{I}}$ and $\boldsymbol{u}^{\mathrm{S}}=\boldsymbol{u}-\boldsymbol{u}^{\mathrm{I}}$ must be absorbed here.

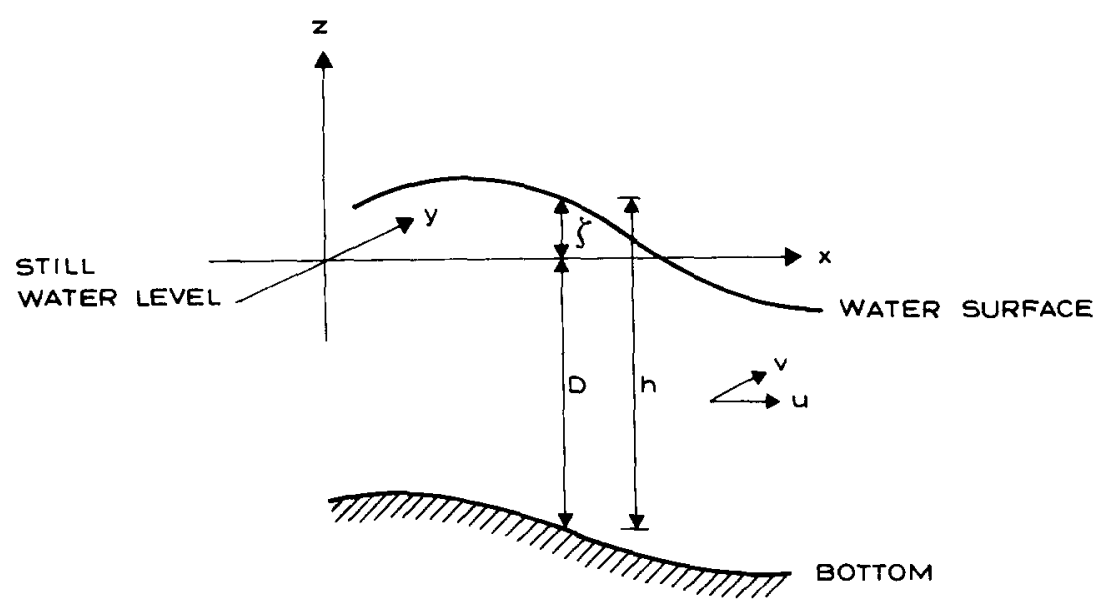

Fig. 1. Definition sketch. 
Previously the method of characteristics was used to separate incoming from outgoing waves. However, this method only works well when the waves are linear and approach the open boundary at almost right angles. In the present paper we propose to generate the waves inside the model boundary by perturbing the surface elevation along a straight line.

We want to generate an incident wave of celerity $c$ and elevation $\eta^{\mathrm{I}}$ that propagates at an angle $\theta$ with respect to the line of generation, $l$. This wave has the celerity $c \sin \theta$ perpendicular to $l$. For simplicity, let $l$ pass through the grid points (if this is not so, the amount to be added is distributed to the grid points closest to $l$ ) and let the distance between successive points on $l$ be $\Delta s$, then the volume flux across $l$ is $\zeta^{\mathrm{I}} c \sin \theta \Delta s$ in both directions. This amount must be balanced by adding volume to the system. Since a grid point covers an area of $\Delta x \Delta y$, where $\Delta x$ and $\Delta y$ are the grid spacing in the $x$ - and $y$-directions, respectively, the surface elevation to be added to $\zeta$ at each grid point of $l$ is:

$\zeta^{\star}=2 \eta^{\mathrm{I}} c \Delta t \frac{\Delta s}{\Delta x \Delta y} \sin \theta$

In one dimension, eq. 3 reduces to:

$\zeta^{\star}=2 \zeta^{\mathrm{I}} \mathrm{Cr}$

where $C r=c \Delta t / \Delta x$ is the Courant number.

In Fig. 2 we show a one-dimensional computation. The left-hand boundary is fully reflecting.

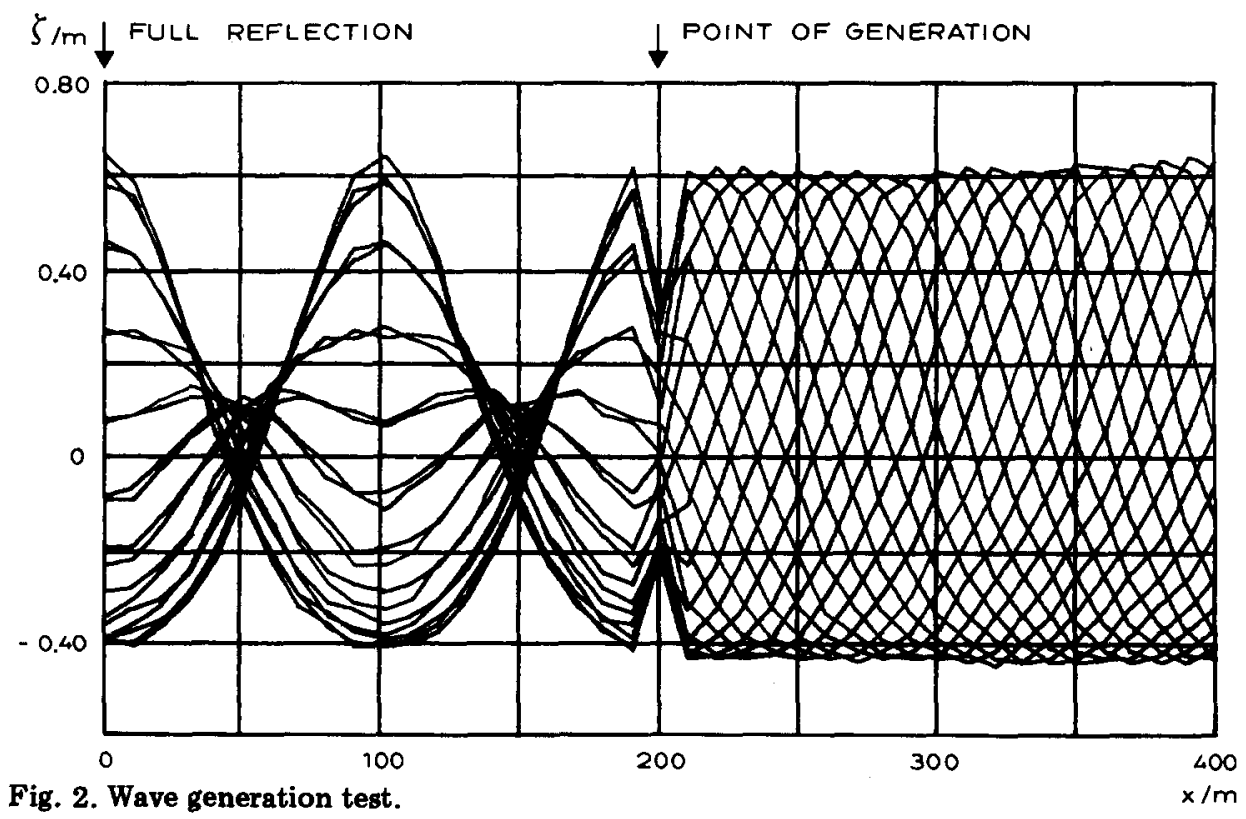


Cnoidal waves of height $0.5 \mathrm{~m}$ are generated at $x=200 \mathrm{~m}$. The water depth is $10 \mathrm{~m}$ and the wave period is $20 \mathrm{~s}$. The surface elevation is plotted each second between 100 and $120 \mathrm{~s}$ after the start of the calculation. We see a standing wave to the left of the line of generation and a progressing wave of double the incident wave height to the right of this line. Since the waves are non-linear we do not have perfect nodal points. The figure shows that the waves, which are reflected at the left boundary, pass through the line of generation without distortion.

\section{ABSORPTION}

By generating waves inside the computational domain we are certain that all waves are outgoing waves. Hence all waves must be absorbed at an open boundary. In the code we do this by dividing, at each time step, the surface elevation and the flow on a few grid lines next to the boundary by a set of numbers which increase towards the boundary. We linearize the Boussinesq eqs. 1 and 2 to get the one-dimensional long-wave equations:

$\frac{\partial \zeta}{\partial t}+\frac{\partial p}{\partial x}=0$

and

$\frac{\partial p}{\partial t}+g D \frac{\partial \zeta}{\partial x}=0$

where $p=u D$ is the flux. We use the modified Preissmann box scheme (McCowan, 1978) in the analysis. This scheme is fully centered on $\left[\left(j+\frac{1}{2}\right) \Delta x, n \Delta t\right]$ in both space and time. We have used the representation:

$$
\begin{aligned}
\left(\zeta_{j+1}^{n+1}-\zeta_{j+1}^{n-1}+\zeta_{j}^{n+1}-\zeta_{j}^{n-1}\right) / 4 \Delta t= & -\left[\alpha\left(p_{j+1}^{n+1}-p_{j}^{n+1}\right)+(1-2 \alpha)\left(p_{j+1}^{n}-p_{j}^{n}\right)\right. \\
& \left.+\alpha\left(p_{j+1}^{n-1}-p_{j}^{n-1}\right)\right] / \Delta x \\
\left(p_{j+1}^{n+1}-p_{j+1}^{n-1}+p_{j}^{n+1}-p_{j}^{n-1}\right) / 4 \Delta t= & -g D\left[\alpha\left(\zeta_{j+1}^{n+1}-\zeta_{j}^{n+1}\right)+(1-2 \alpha)\left(\zeta_{j+1}^{n}-\zeta_{j}^{n}\right)\right. \\
& \left.+\alpha\left(\zeta_{j+1}^{n-1}-\zeta_{j}^{n-1}\right)\right] / \Delta x
\end{aligned}
$$

where $\zeta_{j}^{n}=\zeta(j \Delta x, n \Delta t)$, etc., and $\alpha$ is a weighting factor. After each time step we divide the surface elevation and the fluxes with $\mu(x)$ on the sponge layer grid lines. In eqs. 7 and 8 this corresponds to replacing the variables at time step $n$ and $n-1$ with the values that result after division by $\mu$ and $\mu^{2}$, respectively. Denoting these values by a star we find the differential equations corresponding to the combined process of solving eqs. 7 and 8 and dividing on the grid lines:

$\frac{\partial \zeta^{\star}}{\partial t}+\frac{\partial \nu p^{\star}}{\partial x}=-\frac{1-\mu^{-2}}{\Delta t} \zeta^{\star}$ 
and

$\frac{\partial p^{\star}}{\partial t}+g D \frac{\partial \nu \zeta^{\star}}{\partial x}=-\frac{1-\mu^{-2}}{\Delta t} p^{\star}$

where

$\nu(x)=\alpha+(1-2 \alpha) / \mu+\alpha / \mu^{2}$

Introducing:

$\zeta^{\star \star}=\zeta^{\star} \nu \exp (-i \omega t) / \sqrt{g D}$

and

$p^{\star \star}=p^{\star} \nu \exp (-\mathrm{i} \omega t)$

where we have assumed time-harmonic motion of frequency $\omega$, into eqs. 9 and 10 we finally get the equations:

$\frac{\mathrm{d} \zeta^{\star \star}}{\mathrm{d} x}=-\gamma p^{\star \star}$

and

$\frac{\mathrm{d} p^{\star \star}}{\mathrm{d} x}=-\gamma \zeta^{\star \star}$

where

$\gamma=\left[\mathrm{i} k-\left(\mu^{-2}-1\right) / C r \Delta x\right] / \nu$

in which $C r=\sqrt{g D} \Delta t / \Delta x$ is the Courant number and $k=\omega / \sqrt{g} D$ is the wave number. The sponge layer is the interval $0 \leqq x \leqq x_{\mathrm{s}}$. To be specific we use the function:

$\mu(x)= \begin{cases}\exp \left[\left(2^{-x / \Delta x}-2^{-x_{\mathrm{s}} / \Delta x}\right) \ln a\right] & \text { for } 0 \leqslant x \leqslant x_{\mathrm{s}} \\ 1 & \text { for } x_{\mathrm{s}}<x\end{cases}$

where $a$ is a constant that depends on the number of grid lines in the layer, $x_{\mathrm{s}} / \Delta x$. This function is shown in Fig. 3 for two different sets of values for $x_{\mathrm{s}} / \Delta x$ and $a$. The function $\mu$ is continuous at $x=x_{\mathrm{s}}$.

For $x>x_{\mathrm{s}}$ we write the solution as:

$\zeta^{\star \star}(x)=\exp (\mathrm{i} k x)+R \exp (-\mathrm{i} k x)$

which represents the superposition of an incoming wave from infinity with unit amplitude and a reflected wave with amplitude $R$. Denoting the solution 


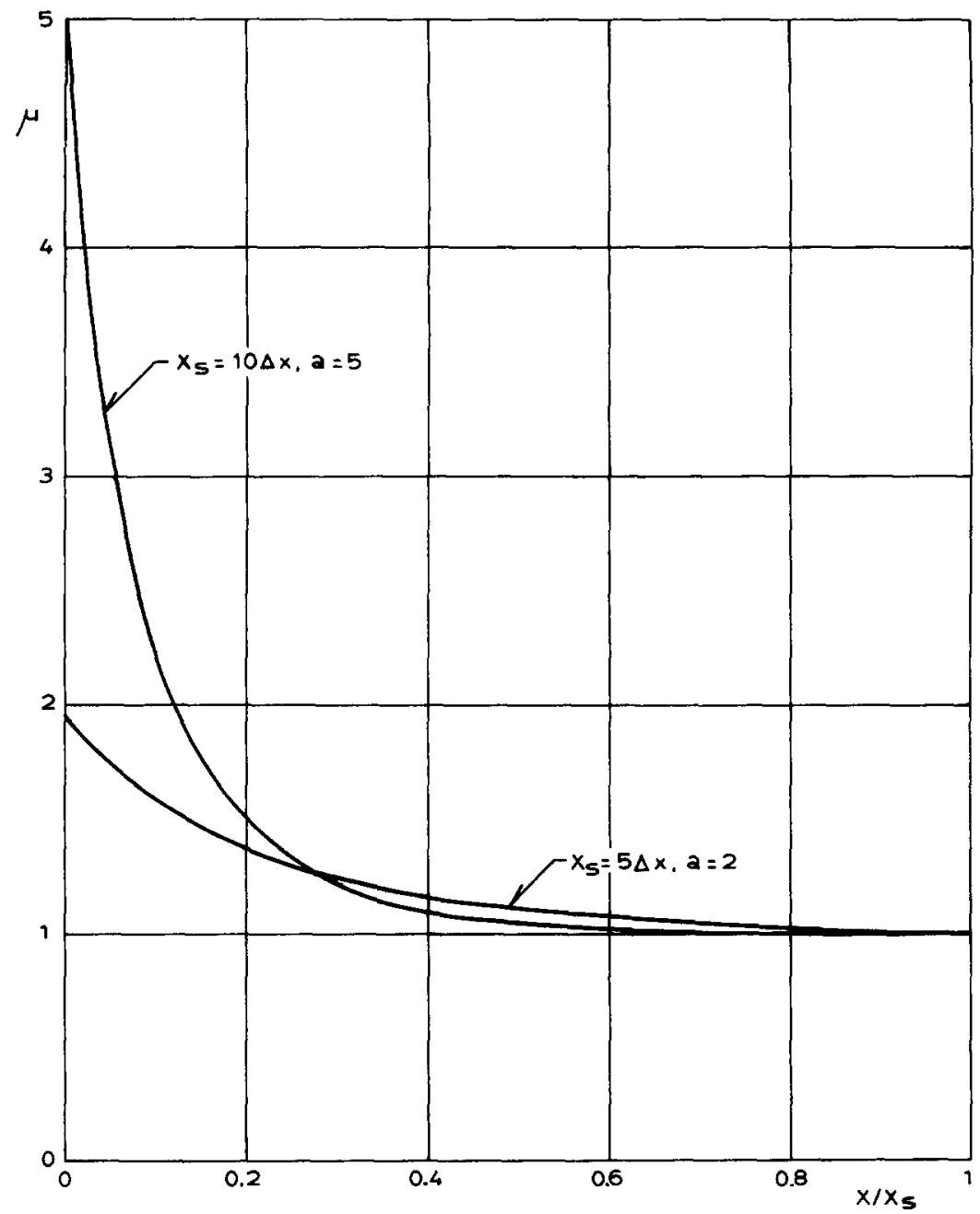

Fig. 3. The function $\mu$ (eq. 15) vs, distance for two different sponge layers.

in the sponge layer by $\zeta_{\mathrm{s}}$ we find the reflection coefficient by matching the two solutions at $x=x_{\mathrm{s}}$. The result is

$R=\frac{\mathrm{i} k \zeta_{\mathrm{s}}-\mathrm{d} \zeta_{\mathrm{s}} / \mathrm{d} x}{\mathrm{i} k \zeta_{\mathrm{s}}+\mathrm{d} \zeta_{\mathrm{s}} / \mathrm{d} x} \mid \underset{\substack{x=x_{\mathrm{s}} \\ \exp \left(-2 \mathrm{i} k x_{\mathrm{s}}\right)}}{\operatorname{ex}}$

At the boundary $x=0$ we impose the condition $\xi=0$. The solution in the sponge layer is then given by:

$\zeta_{\mathrm{s}}=A\left[\exp \left(\int_{0}^{x} \gamma(s) \mathrm{d} s\right)-\exp \left(-\int_{0}^{x} \gamma(s) \mathrm{d} s\right)\right] / \nu$

where $A$ is a constant. 


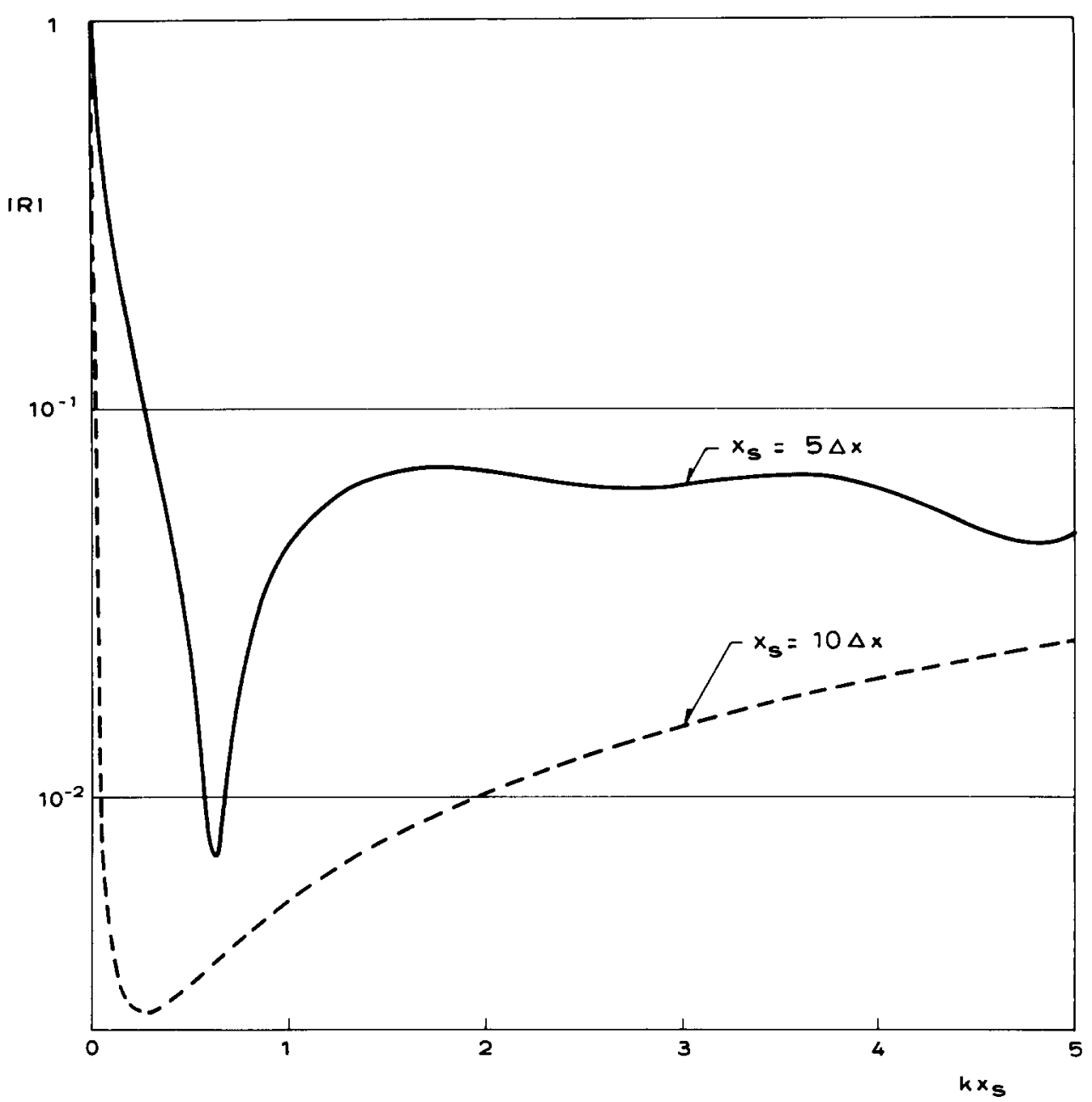

Fig. 4. Reflection coefficient vs. the dimensionless wave number $k x_{\mathrm{s}}$ for two different numbers of grid points in the "sponge" layer.

In Fig. 4 we have plotted $|R|$ against the non-dimensional wave number $k x_{s}$, which is $2 \pi$ times the number of wave lengths in the sponge layer corresponding to $\alpha=0.5$. For a layer of thickness $x_{\mathrm{s}}=5 \Delta x$ we choose $a=2$ and for a layer of thickness $x_{8}=10 \Delta x$ we choose $a=5$ in eq. 15 . The use of only five grid lines in the sponge layer covers most engineering applications. In Fig. 5 we have shown how the sponge layer behaves in a one-dimensional simulation. Sinewaves of a wavelength of $200 \mathrm{~m}$ are created on the left-hand boundary. At the right-hand boundary, waves are absorbed in a sponge layer of width $x_{8}=5 \Delta x$.

The maximum and minimum wave heights are $H_{\max }=0.044$ and $H_{\min }=$ 0.040 , respectively. Hence, the reflection coefficient is:

$|R|=\frac{H_{\max }-H_{\min }}{H_{\max }+H_{\min }} \simeq 5 \%$ 


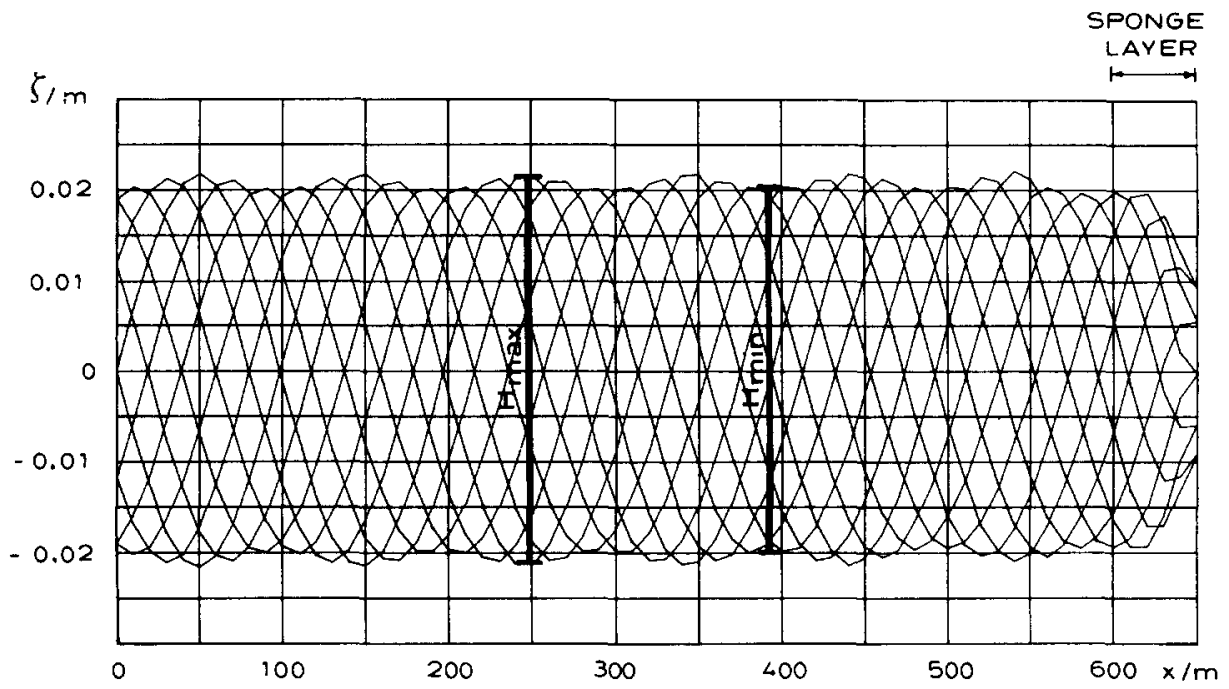

Fig. 5. Wave absorption test.

For this example $k x_{\mathrm{s}}=\pi / 2$ and eq. 18 agrees fairly well with the analytic result of $|R|=6.8 \%$. We have tried the sponge layer with solitary waves of the same height as the water depths, and the reflected waves could not be separated from the trailing noise of this high wave. In order to visualize the sponge layer effect, we have arranged two sponge layers, each of width $5 \Delta x$, symmetrical about the line $x=250$, as shown in Fig. 6 . At the left boundary

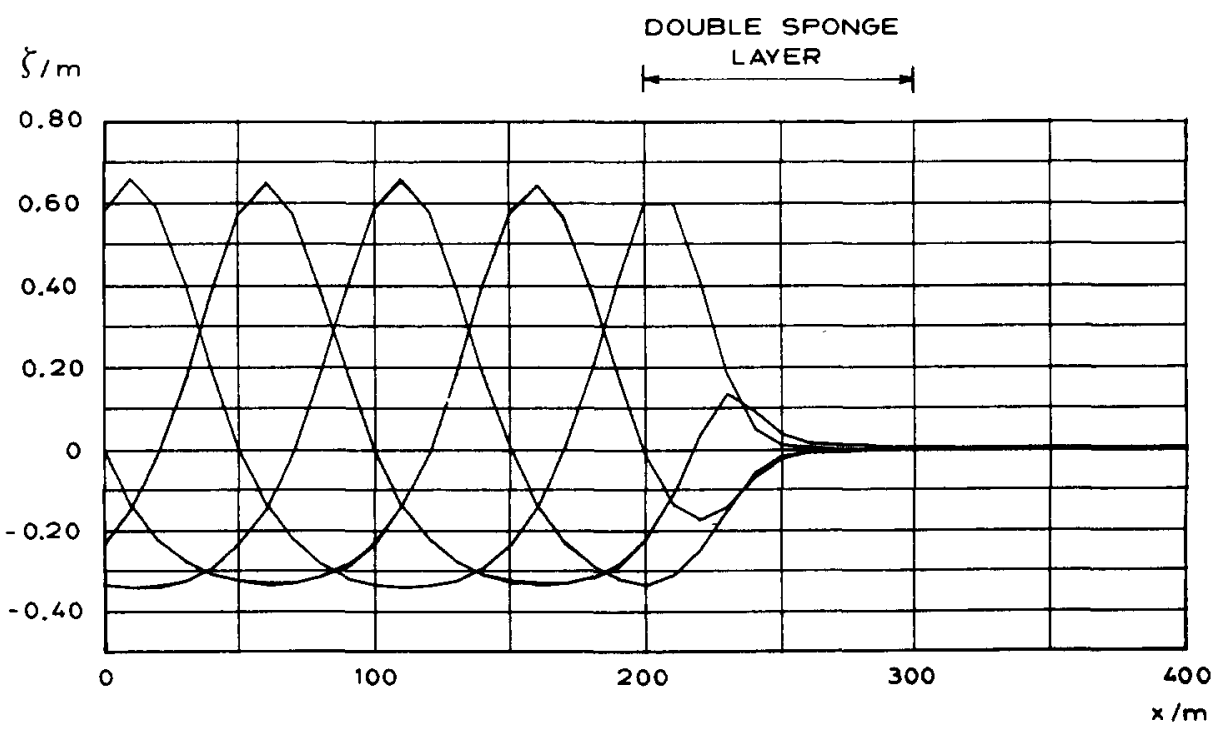

Fig. 6. Wave absorption test.

Fig. 7. Wave diffraction test with internal wave generation. a. Contour plot of surface elevation. $\mathrm{b}$. Velocity plot. $t=75$ sec. 


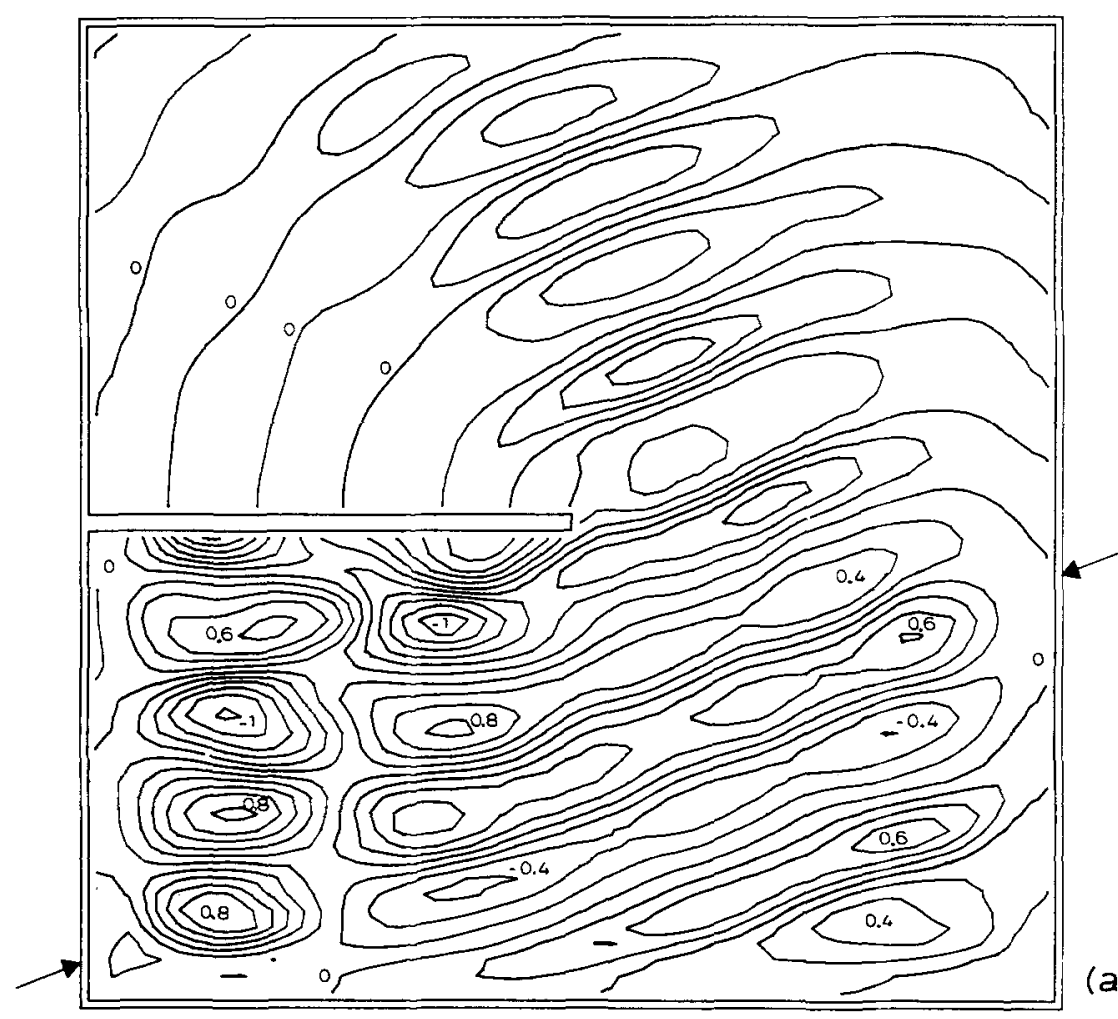

293

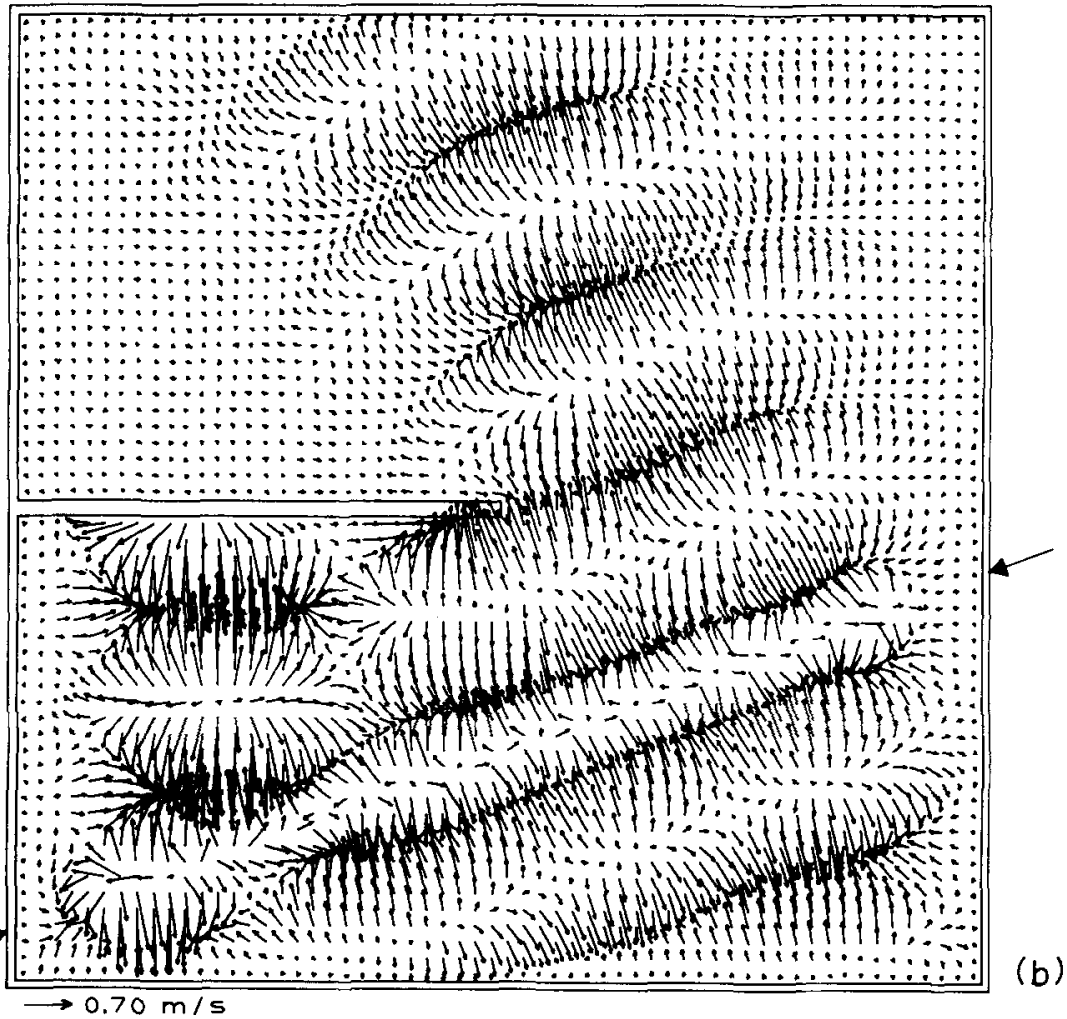


we generate cnoidal waves of one meter wave height. The grid spacing is $\Delta x=10 \mathrm{~m}$. The surface elevation is hardly visible after the double passage through the sponge layer.

\section{TWO-DIMENSIONAL SIMULATION RESULTS}

In the two-dimensional simulation we use the S21MK8 system (Abbott, 1979). For all the examples we show in this section, we use a square model with a side length of $600 \mathrm{~m}$. The still water depth is $9 \mathrm{~m}$. We use a square grid with $\Delta x=10 \mathrm{~m}$. All boundaries are open and we use sponge layers of width $5 \Delta x$ here. The lines of wave generation are each marked by two arrows on the figures.

In Fig. 7 a simple diffraction test is shown. Figure $7 \mathrm{a}$ shows the surface elevations and Fig. $7 \mathrm{~b}$ shows the velocities $75 \mathrm{~s}$ after the start of the simulation. The breakwater is fully reflecting. Waves are added perpendicular to the line of generation. The incident wave is a sine wave of period $12 \mathrm{~s}$ and height $0.5 \mathrm{~m}$. A standing wave pattern is formed - as expected - in front of the breakwater and this pattern is only insignificantly distorted by the generation process.

In order to show the feasibility of generating a short-crested wave field, i.e. a wave field with a directional spectrum, we have performed the simulations shown in Figs. 8 and 9. In Fig. 8 we show the generation of a sine wave, which propagates obliquely to the grid line, forming an angle of $45^{\circ}$ with the grid line. The period is $12 \mathrm{~s}$ and the wave height is $0.5 \mathrm{~m}$. Figure $8 \mathrm{a}$ shows a contour plot of the surface elevations and Fig. $8 \mathrm{~b}$ shows the velocity plot $75 \mathrm{a}$ after the start of the simulation. The result of a simulation of the addition of two waves is shown in Fig. 9. Sine waves of period $12 \mathrm{~s}$ are added perpendicular to the lines marked 1 and 2 in the figure. The velocity plot is drawn $36 \mathrm{~s}$ after the start of the computations. The short crestedness of the waves is apparent.

From Fig. 8 we see that waves of oblique directions can be generated on a line parallel to the model boundary. Hence the additional computational points, which must be introduced to accommodate the sponge layer, are five times the number of grid points along the open boundaries. Higher-order boundary conditions require a similar amount of additional grid points (cf. Engquist and Majda, 1977); but the present method, which appears to be generally applicable, is by far the simplest to implement.

\section{CONCLUSIONS}

A new way of implementing radiation boundary conditions in finite difference schemes is reported. The incident waves are simply added on lines inside the model boundary and then all waves are absorbed on the boundary.

Fig. 8. Generation of waves which propagate in a direction forming an angle of $45^{\circ}$ with the line of generation. a. Contour plot of surface elevation. b. Velocity plot. $t=75$ sec. 


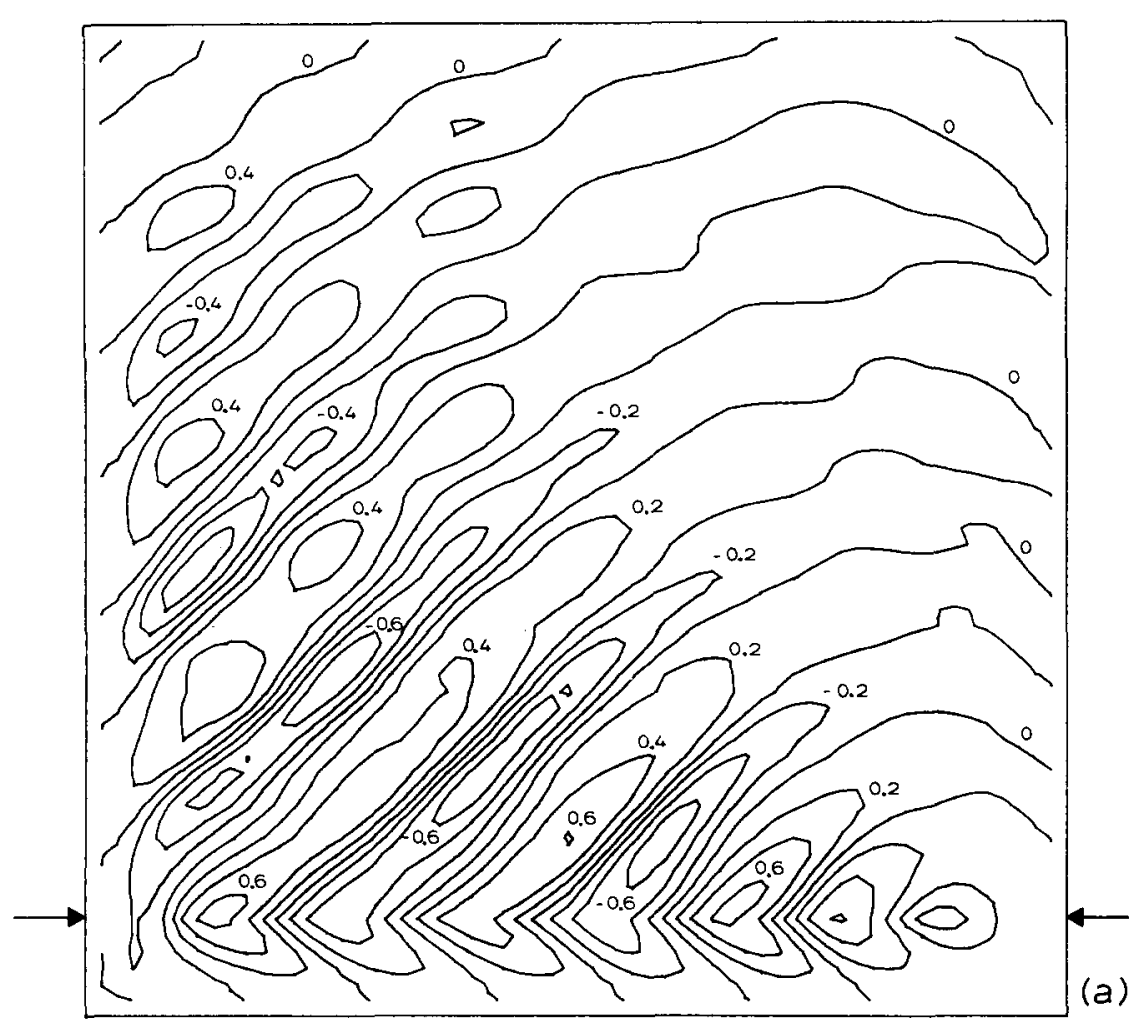

295

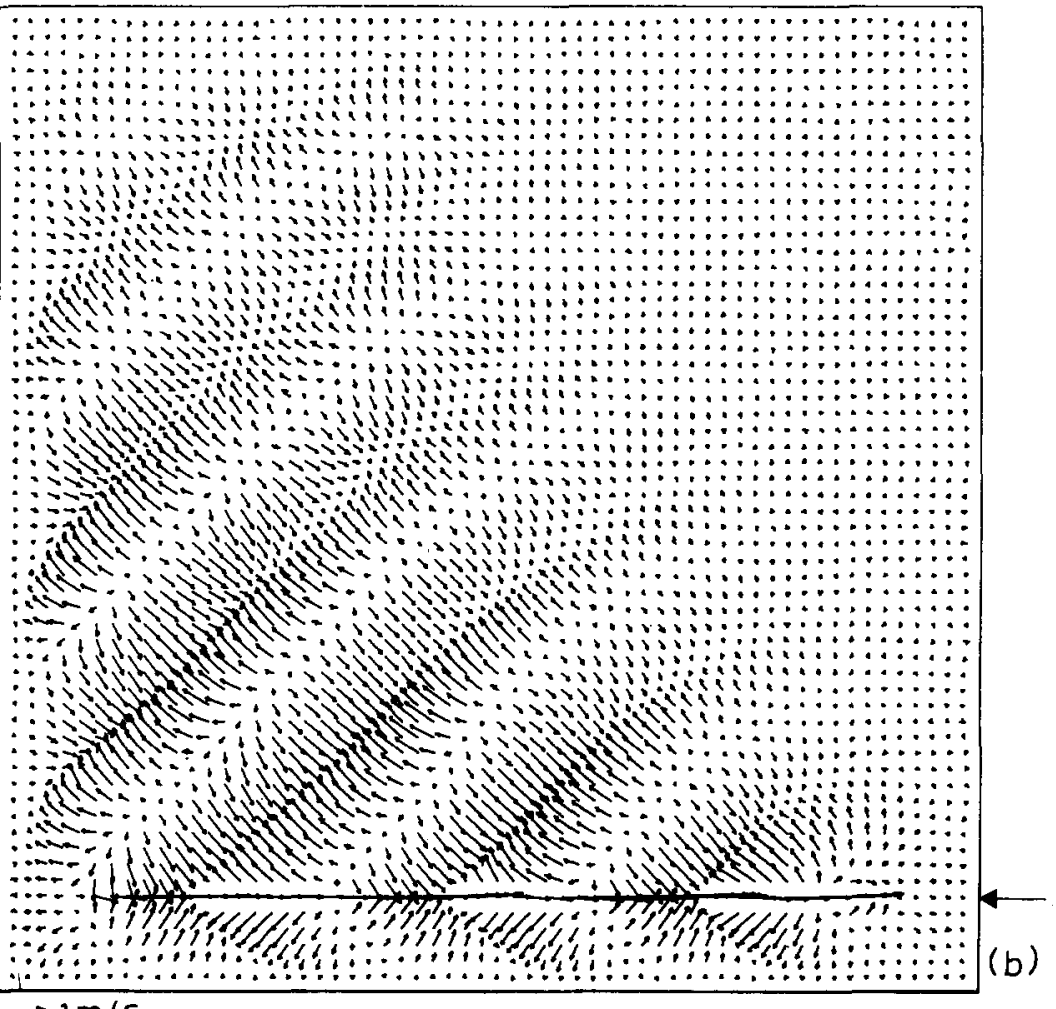




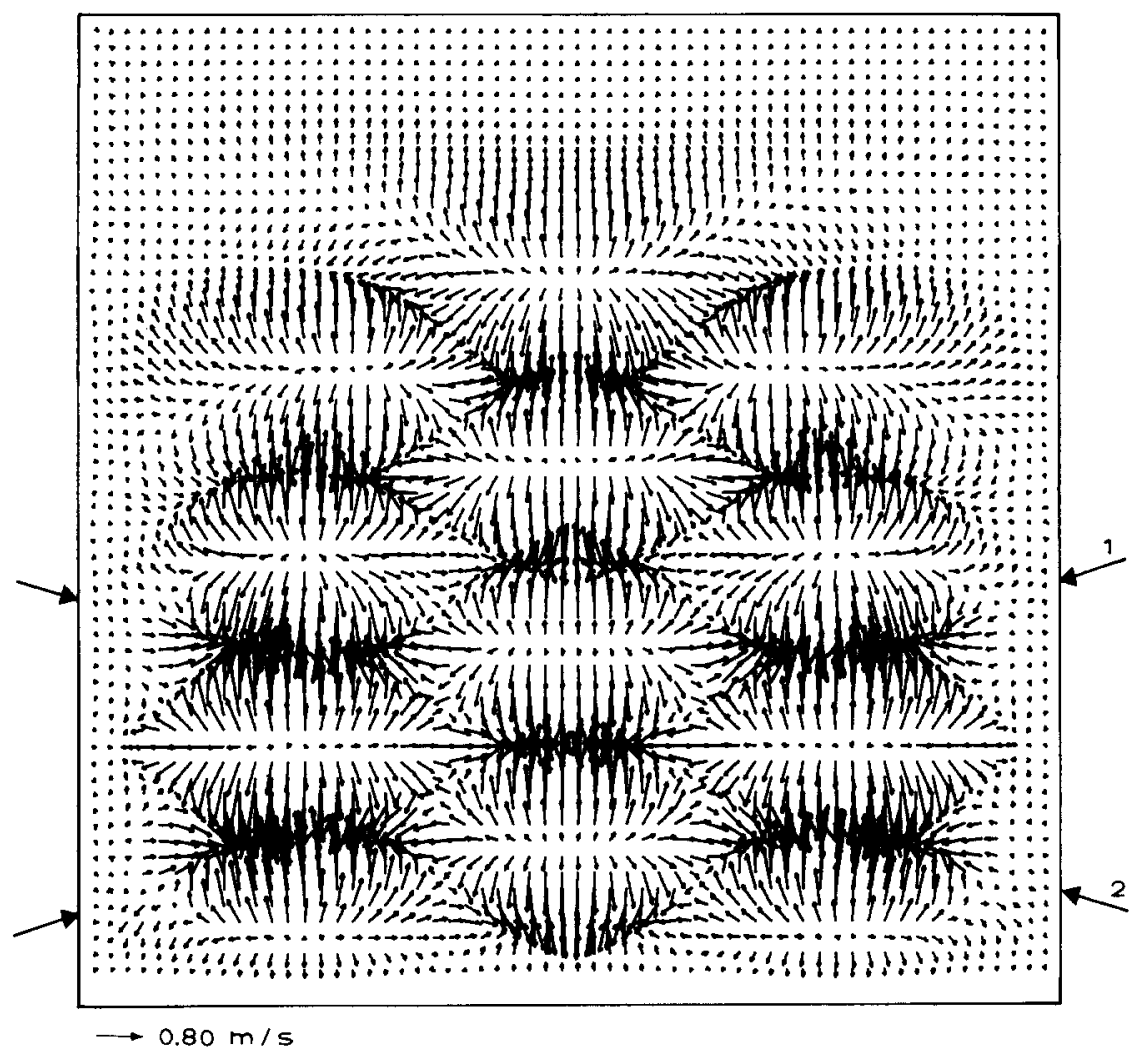

Fig. 9. Generation of short crested waves. Velocity plot. $t=36$ sec.

The linear analysis for the sponge layers shows that they have very broadbanded damping characteristics. Using only five grid lines in the sponge layer, reflection coefficients of less than $7 \%$ magnitude were obtained even with only $5 \%$ of the wave length in the sponge layer. The simulation results show that general incident wave fields can be generated by the new method and that the scattered field is only insignificantly distorted by the generation process and the sponge layers at the boundary.

\section{REFERENCES}

Abbott, M.B., 1979. Computational Hydraulics. Pitman, London, 326 pp.

Abbott, M.B., Petersen, H.M. and Skovgaard, O., 1978. On the numerical modelling of short waves in shallow water. J. Hydraul. Res., 16: 173-204.

Engquist, B. and Majda, A., 1977. Absorbing Boundary Conditions for the Numerical Simulation of Waves. Math. Comp., 31: 629-651.

Israeli, M. and Orszag, S.A., 1981. Approximation of Radiation Boundary Conditions. J. Comp. Phys., 41: 115-131. 
Madsen, P.A., 1983. Wave reflection from a vertical permeable wave absorber. Coastal Eng., in prep.

McCowan, A.D., 1978. Numerical simulation of shallow water waves. Fourth Australian Conference on Coastal \& Ocean Engineering, Adelaide, 8-10 Nov. 1978. Reprints, pp. $132-136$.

Peregrine, D.H., 1967. Long waves on a beach. J. Fluid Mech., 27: 815-827.

Preissmann, A., 1961. Propagation des Intermescences dans les Canaux et Rivières, Congrès du l'Ass, Fr. de Calc., Grenoble.

Verboom, G.K., Stelling, G.S. and Officer, M.J., 1983. Boundary conditions for the shallow water equations. In: M.B. Abbott and J.A. Cunge (Editors), Engineering Applications of Computational Hydraulics I. Pitman, London, pp. 230-262. 